\title{
Ovarian fibrothecoma - a diagnostic dilemma
}

\begin{abstract}
Background: The presentation of ovarian fibrothecoma is highly deceptive and it may be undiagnosed till histopathology reveals the actual diagnosis. Hence, the clinician must be aware of such cases which may present as a diagnostic dilemma.

Introduction: Ovarian fibrothecomas are rare ovarian neoplasm. We report a case where clinical presentation was highly deceptive and suggestive of malignant tumor. However, ascitic fluid cytology revealed absent malignant cells. On histopathological examination, it was diagnosed as benign fibrothecoma with cystic changes. Postoperative follow-up for about six months was uneventful.

Case: A 45 year old female presented with large abdominal lump of 20 weeks size associated with pain abdomen. She was admitted for management and evaluation. Hematological and biochemical parameters were normal. USG revealed a large multilocular, predominantly cystic lesion $20.9 \times 9.6 \times 11.4 \mathrm{~cm}$ in pelvis. CECT revealed ovarian cystadenocarcinoma left ovary with locoregional mass effect, mild ascites and suspicious metastasis to internal iliac lymph nodes. Hence panhysterectomy and omentectomy was performed as radiological and preoperative clinical diagnosis was malignant ovarian tumor. On gross examination, a well encapsulated, multinodular cystic tumor of left ovary about $17 \times 14 \times 7 \mathrm{~cm}$ was identified. Cut surface was mostly solid with few cystic areas. On microscopic examination, multiple sections showed spindle shaped cells in storiform and palisading pattern. No mitotic activity was identified. On special staining, it was positive for vimentin. Hence, final diagnosis came to be as benign fibrothecoma of ovary
\end{abstract}

Conclusion: The accurate preoperative diagnosis of ovarian fibrothecoma with cystic changes could have prevented the extensive surgical intervention such as bilateral salpingo- oopherectomy with hysterectomy.

Keywords: ovarian fibrothecoma, ascites, tumor
Volume 10 Issue 3 - 2019

\author{
Nikita Kumari,' Bindu Bajaj² \\ 'Attending Consultant at Sitaram Bhartia Institute of Science \\ and Research, Ex Senior Resident at VMMC and Safdarjung \\ Hospital, India \\ ${ }^{2}$ Associate Professor at VMMC and Safdarjung Hospital, New \\ Delhi, India
}

\begin{abstract}
Correspondence: Nikita Kumari, Attending Consultant at Sitaram Bhartia Institute of Science and Research, Ex Senior Resident atVMMC and Safdarjung Hospital, New Delhi, India, Tel 9654251653, Email drnikitakumari@gmail.com
\end{abstract}

Received: May 27, 2019| Published: June 13, 2019

\section{Introduction}

Fibrothecomas of ovary constitute about $4 \%$ of all ovarian tumors. They represent a group of benign tumors arising in stroma and exhibit a morphological spectrum consisting of entirely fibroblasts and producing collagen (Fibromas) to those containing more plump spindle cells with lipid droplets (Thecomas). When a tumor contains mixture of these cells, they are termed as fibrothecomas. . Also, they have been reported to show myxoid change and degeneration. These tumors occur at all ages, but are more frequent during middle age. ${ }^{1}$

We report a case of ovarian mass with abdominal distension whose clinical presentation was highly deceptive and was clinically and radiologically diagnosed as malignant ovarian tumor. Hence the patient had to undergo complete staging laprotomy. MRI was not used as an imaging modality in this case which could have otherwise suggested fibrothecoma as a differential diagnosis.

\section{Case summary}

A 45 year old female presented to gynecology emergency with large abdominal lump of 20 weeks size with pain lower abdomen. Menstrual history was normal. General physical and systemic examination was normal. Local examination of the abdomen and pelvis revealed a hard mass of $15 \times 10 \mathrm{~cm}$ in midline arising from pelvis. Mild ascites was present. Hematological and biochemical parameters were within normal limits. Ca 125 was $21.3 \mathrm{U} / \mathrm{ml}$.

Ultrasonography revealed a large multilocular, predominantly cystic lesion of $20.9 \times 9.6 \times 11.4 \mathrm{~cm}$ in pelvis with multiple retroperitoneal lymph nodes. B/L ovaries were not seen separately. CECT revealed ovarian cystadencarcinoma left ovary with locoregional mass effect, mild ascites and suspicious metastasis to internal iliac lymph nodes. Since radiological and preoperative clinical diagnosis was malignant ovarian tumor, the patient underwent total abdominal hysterectomy with $\mathrm{B} / \mathrm{L}$ salpingo-oopherectomy and omentectomy.

On gross examination, a well encapsulated, multinodular cystic tumor of left ovary about $17 \times 14 \times 7 \mathrm{~cm}$ was identified (Figure 1). Cut surface was mostly solid with few cystic areas. On cut section hemorrhagic fluid came out (Figure 2). Uterus, cervix, right ovary and both tubes were unremarkable.

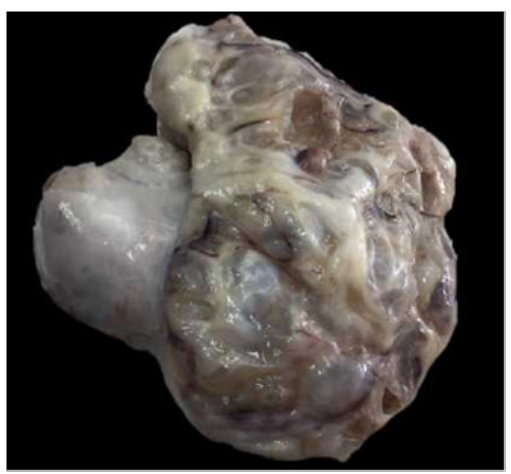

Figure I Multinodular cystic tumor of left ovary. 


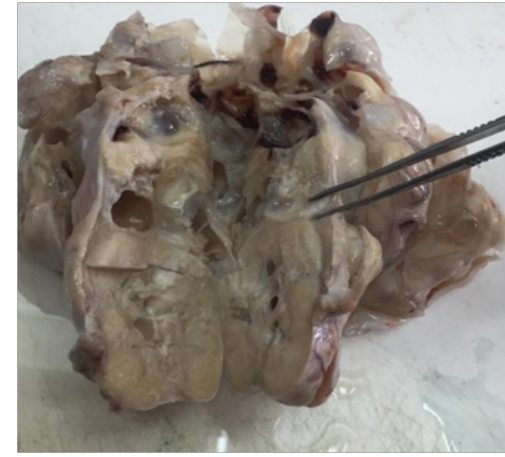

Figure 2 Cut section hemorrhagic fluid.

On microscopic examination, multiple sections showed spindle shaped cells arranged in storiform and palisading pattern. The cells were monomorphic with central nucleus. No mitotic activity could be identified (Figures 3-5).

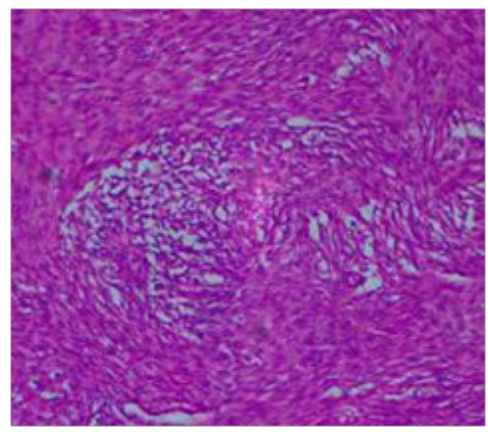

Figure 3 Multiple sections showed spindle shaped cells arranged in storiform pattern.

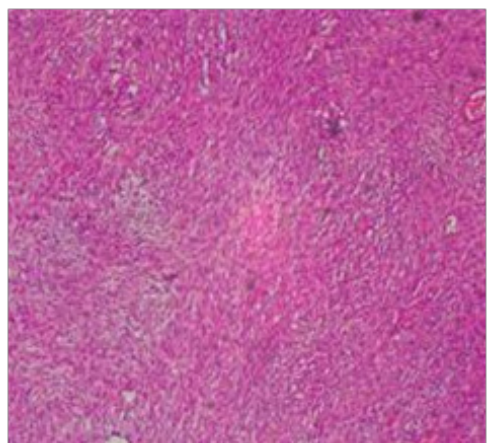

Figure 4 Multiple sections showed spindle shaped cells arranged in palisading pattern.

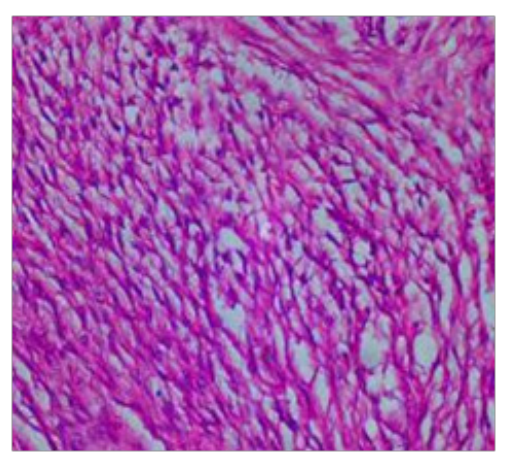

Figure 5 Cells were monomorphic with central nucleus.
Tumor cells were diffusely positive for oil red O (Figure 6).

On special staining, it was positive for Vimentin, which is a characteristic feature of ovarian fibrothecoma (Figure 7).

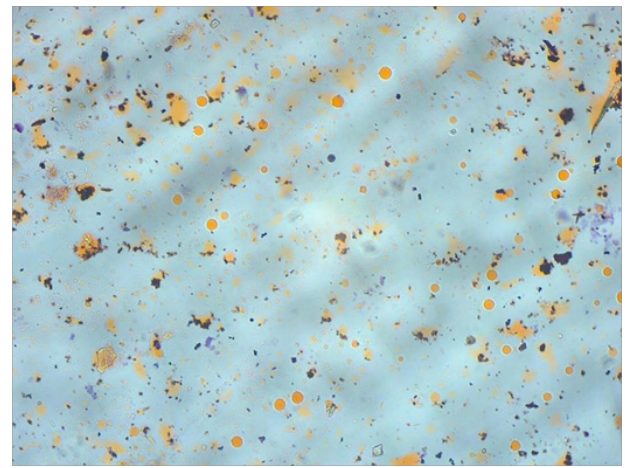

Figure 6 Tumor cells were diffusely positive for oil red $\mathrm{O}$.

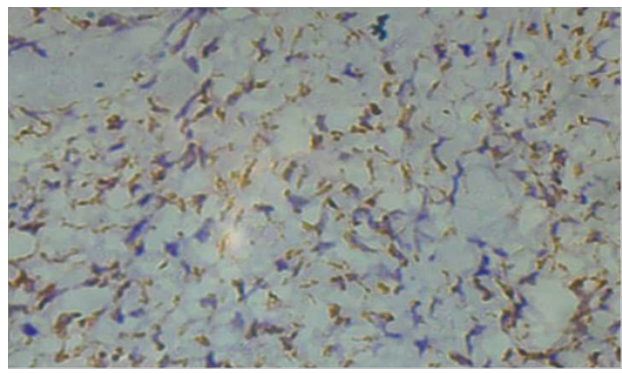

Figure 7 Characteristic feature of ovarian fibrothecoma.

Histopathology revealed as fibrothecoma left ovary. The cytology of ascitic fluid and omental biopsy was negative for malignancy. Postoperative course was uneventful. The patient is well and asymptomatic 6 months after surgery.

\section{Discussion}

Fibrothecoma are of gonadal stromal cell origin accounting for $3-4 \%$ of all ovarian tumors. ${ }^{2,3}$ They are unilateral ${ }^{3}$ in about $90 \%$ cases and are rarely malignant. ${ }^{4,5}$ The clinical presentation may be nonspecific such as pelvic and abdominal pain or distension, which was there in our case. It may be accompanied by Meig's syndrome (ovarian fibroma, hydrothorax and ascites) and Basal cell nevus syndrome (bilateral ovarian fibroma, multiple basal cell carcinoma of skin, odontogenic keratocysts, etc). Our case diagnosed as fibrothecoma was associated with ascites only.

Some ovarian thecomas may be hormonally active and show estrogenic activity such as menstrual irregularities, amenorrhea, endometrial hyperplasia and endometrial carcinoma. In the present case no such abnormality was there. Grossly, fibrothecomas are usually round, oval or lobulated encapsulated hard gray white masses covered by intact ovarian serosa. Edema and cystic degeneration are relatively common ${ }^{2,4}$ which was there in the present case. Samanth et al. ${ }^{6}$ reported that fibrothecoma $>10 \mathrm{~cm}$ tend to be associated with myxoid change or degeneration. Also, discrepancy between arterial and venous and lymphatic drainage could lead to stromal edema. ${ }^{6}$

On CT scan, ovarian fibrothecomas may appear as a homogenous solid tumor with varying degrees of enhancement. ${ }^{7}$ In $79 \%$ of the cases the tumor appears as a solid mass with delayed accumulation 
of contrast medium, while in $21 \%$ of the cases the tumor is partly or mainly cystic thus making differential diagnosis from other ovarian masses, such as serous cystadenofibromas or even malignant tumors, difficult. ${ }^{8}$ The absence of arterial vessels and the absence or slight early uptake of contrast enhancement are characteristic signs and may be useful in considering the diagnosis of a fibrothecoma preoperatively. ${ }^{8}$

On MRI, fibrothecomas typically show predominantly low signal intensity on T2 weighted images. ${ }^{2}$ Also, scattered high signal areas may be present representing areas of cystic degeneration $+/$ - oedema.

Differential diagnosis of fibrothecomas includes pedunculated and intraligamentous leiomyomas and other solid ovarian masses such as Brenner tumors, granulosa cell tumors and dysgerminomas. ${ }^{2,4,9}$ In the presence of extensive cystic degeneration, the fibrothecoma can be easily mistaken for a malignant ovarian tumor. ${ }^{9}$ Thus, an accurate preoperative diagnosis by MRI may prevent an excessive surgical intervention, especially if the patient is in younger age group.

\section{Conclusion}

Ovarian fibrothecomas represent an ovarian stromal neoplasm developing in a wide spectrum of clinical settings. Probably it is the most inaccurately diagnosed lesion of the female gonad, clinically as well as histologically.

Early diagnosis and surgical resection is the treatment of choice for ovarian fibrothecomas. The accurate preoperative diagnosis of ovarian fibrothecomas with cystic degeneration could have prevented the extensive surgical intervention such as bilateral salpingooophorectomy with hysterectomy. Tumorectomy is indicated for young patients. Radical surgery in terms of bilateral salpingooophorectomy is indicated for perimenopausal and menopausal patients and is associated with a good prognosis.

\section{Conflicts of interest}

Author has no conflict of interest to declare.

\section{References}

1. Scully RE, Youg RH, Clement PB. Tumors of the ovary, maldeveloped gonads, fallopian tube and broad ligament. International Journal of Gynecological Pathology. 1999;18(3):288.

2. Troiano RN, Lazzarini KM, Scoutt LM, et al. Fibroma and fibrothecoma of the ovary: MR imaging findings. Radiology. 1997;204(3):795-798.

3. Chechia A, Attia L, Temime RB, et al. Incidence, clinical analysis, and management of ovarian fibromas and fibrothecomas. Am J Obstet Gynecol. 2008;199(5):473.e1-e4.

4. Imaoka I, Wada A, Kaji Y, et al. Developing an MR imaging strategy for diagnosis of ovarian masses. Radiographics. 2006;26(5):1431-1448.

5. Liu H, Hao SH, Li WM. Giant malignant ovarian fibrothecoma involved with retroperitoneal structures mimicking a retroperitoneal sarcoma. Arch Gynecol Obstet. 2009;279(5):763-765.

6. Samanth KK, Black WC. Benign ovarian stromal tumors associated with free peritoneal fluid. Am J Obstet Gynecol. 1970;107(4):538-545.

7. Mak CW, Tzeng WS, Chen CY. Computed tomography appearance of ovarian fibrothecomas with and without torsion. Acta Radiol. 2009;50(5):570-575.

8. Bazot M, Ghossain MA, Buy JN, et al. Fibrothecomas of the ovary: CT and US findings. J Comput Assist Tomogr. 1993;17(5):754-759.

9. Takeshita T, Shima H, Oishi S, et al. Ovarian fibroma (fibrothecoma) with extensive cystic degeneration: unusual MR imaging findings in two cases. Radiat Med. 2005;23(1):70-74.

\section{Acknowledgments}

None. 